\title{
1 Signal complexity indicators of health status in clinical-EEG
}

2 Kelly Shen ${ }^{1 *}$, Alison McFadden ${ }^{1}$, Anthony R. McIntosh ${ }^{1,2}$

$3 \quad{ }^{1}$ Rotman Research Institute, Baycrest Centre

4 2University of Toronto

$5 \quad{ }^{*}$ Corresponding Author

6 Email: kshen@research.baycrest.org

7 Mailing Address:

83560 Bathurst Street

9 Toronto ON M6A 2E1

10 Canada 


\section{Abstract}

12 Brain signal variability changes across the lifespan in both health and disease, likely

13 reflecting changes in information processing capacity related to development, aging and

14 neurological disorders. While signal complexity, and multiscale entropy (MSE) in particular,

15 has been proposed as a biomarker for neurological disorders, most observations of altered

16 signal complexity have come from studies comparing patients with few to no comorbidities

17 against healthy controls. In this study, we examined whether MSE of brain signals was

18 distinguishable across individuals in a large and heterogeneous set of clinical-EEG data.

19 Using a multivariate analysis, we found unique timescale-dependent differences in MSE

20 across various neurological disorders. We also found MSE to differentiate individuals with

21 non-brain comorbidities, suggesting that MSE is sensitive to brain signal changes brought

22 about by metabolic and other non-brain disorders. Such changes were not detectable in the

23 spectral power density of brain signals. Our findings suggest that brain signal complexity

24 may offer complementary information to spectral power about an individual's health status

25 and is a promising avenue for clinical biomarker development.

26 Keywords: Multi-scale entropy, EEG, neurodegenerative disease, epilepsy 


\section{Introduction}

28 A growing literature suggests that some degree of brain signal variability is vital to optimal

29 brain function. Although seemingly paradoxical, noisy (or complex) brain signals are related

30 to a greater capacity for information processing as compared to more predictable signals

31 (Garrett et al., 2018; Vakorin and McIntosh, 2012). Sample entropy is one way to capture the

32 variability of a brain signal (Richman and Moorman, 2000) and multiscale entropy (MSE),

33 where complexity is examined across multiple timescales (Costa et al., 2005), has been

34 particularly useful in broadening our understanding of the role of noise in brain health and

35 disease. MSE, like other measures of entropy, captures the variability in a signal but can

36 additionally differentiate variability induced by increasing randomness, such that white

37 noise gives lower MSE values (Costa et al., 2005). An increase in MSE has been observed in

38 tasks requiring memory retrieval (Heisz et al., 2012) or the integration of stimulus features

39 (Misić et al., 2010) and seems to support accurate and stable behavior (Misić et al., 2010;

40 Raja Beharelle et al., 2012). MSE has been shown to have timescale-dependent shifts during

41 brain development (Hasegawa et al., 2018; Lippé et al., 2009; Miskovic et al., 2016;

42 Szostakiwskyj et al., 2017) and aging (McIntosh et al., 2014; Sleimen-Malkoun et al., 2015; H.

43 Wang et al., 2016) that supports cognitive function (Heisz et al., 2015; Yang et al., 2013),

44 reflecting changes in the brain's information processing capacity across the lifespan. MSE

45 also reflects processing capacity changes related to various brain diseases including

46 dementia (Bertrand et al., 2016; Grieder et al., 2018; Niu et al., 2018), neurodevelopmental

47 disorders (Mišić et al., 2015; Takahashi et al., 2016; Weng et al., 2017), and psychiatric

48 disorders (Hager et al., 2017; Takahashi, 2013; Yang et al., 2015). 
In nearly all of these studies, brain signal complexity changes related to various brain

50 diseases have been detected by comparing individuals with few to no comorbidities against

51 matched healthy controls using data collected in highly controlled laboratory environments.

52 While MSE has been proposed for use as a clinical biomarker for various neurological

53 disorders (Jeste et al., 2015; Lu et al., 2015; Tsai et al., 2015), whether differences in brain

54 signal complexity can be detected across individuals of a heterogenous clinical population

55 per se remains unknown. In this study, we leveraged the Temple University Corpus EEG

56 database (Obeid and Picone, 2016) to test the utility of MSE as an indicator of health status

57 in a large and heterogeneous clinical population. We found MSE of clinical-EEG signals

58 differentiated individuals of varying brain disorders. Interestingly, we also found MSE to

59 differentiate between individuals with non-brain comorbidities and those without

60 comorbidities. 


\section{Methods}

\section{Subjects}

63 Clinical EEG data and corresponding physician reports were downloaded from the Temple

64 University Hospital EEG Epilepsy Corpus (v0.0.1) containing 100 subjects deemed to have

65 epilepsy and $\quad 100$ subjects without epilepsy

66 (https://www.isip.piconepress.com/projects/tuh_eeg/) (Obeid and Picone, 2016). Subjects

67 from the epilepsy group were included in our sample if the report indicated a previous

68 diagnosis of epilepsy, if the EEG supported a diagnosis of epilepsy, or if the patient had

69 experienced 2 or more unprovoked seizures occurring more than 24 hours apart and the

70 EEG did not contraindicate epilepsy. Subjects without epilepsy were included if they did not

71 meet any of these criteria. Subjects from either group were excluded if a seizure occurred

72 during the recording, if the subject's level of consciousness was decreased, or if the subject

73 was under the effect of a device likely to cause substantial EEG artifact such as a pacemaker

74 or ventilator. Subjects were also excluded if their recordings were deemed unsuitable in the

75 preprocessing stage due to the presence of artifacts.

76 Demographic and clinical characteristics were extracted from the physician reports

77 (Table 1). For the various brain-acting medications (anti-epileptic drugs, barbiturates,

78 benzodiazepines, antipsychotics, and antidepressants), subjects were considered to be on

79 them if their medication list included at least one medication of that category. The total

80 number of other (i.e., not brain-acting) medications for each subject was computed by

81 counting the number of total medications listed for the subject and subtracting the number

82 of medications that fell into the brain-acting medication categories listed above. If the 
83 medication list stated "others" or a pluralized general category of medications (i.e.

84 "antihypertensives"), two medications were added to the non-brain medication count. Most

85 of the non-brain acting medications reported $(69.3 \% ; 223 / 322)$ are those used to treat

86 cardiovascular disease, diabetes or chronic respiratory illness. Seizure classifications and

87 terms were determined as outlined by the International League Against Epilepsy (Berg et al.,

88 2010; Blume et al., 2001). A subject was considered to have experienced generalized or focal

89 seizures if their physician's report contained either a diagnosis falling in one of those

90 categories or a description of seizures matching the expected presentation for that seizure

91 classification. Thirty-four subjects experienced seizures of unknown classification and were

92 excluded from analysis. A further 3 subjects did not have age or sex information available

93 and were also excluded from analysis. This resulted in a total sample size of 163 subjects.

94 Accepted phrases for stroke included indication of a past or present ischemic stroke,

95 hemorrhagic stroke, "CVA", or intracerebral bleed. Accepted diagnoses for degenerative

96 brain diseases included Alzheimer's disease, Parkinson's disease, and dementia. Accepted

97 diagnoses for psychiatric disorders included anxiety, depression, bipolar disease, and

98 schizophrenia. Accepted diagnoses for neurodevelopmental disorders included Down's

99 syndrome, ADHD, intellectual disabilities, and cerebral palsy. Finally, other brain disorders

100 and injuries included head trauma, brain surgery, brain cancer or metastases, hypoxic brain

101 injuries, encephalitis and meningitis. 
102 Table 1. Demographic and clinical characteristics of study sample.

\begin{tabular}{ll}
\hline Variables & $\begin{array}{l}\text { Subjects } \\
(n=163)\end{array}$ \\
\hline Age, mean (SD, range) & $52.12(19.88,7-91)$ \\
Sex, $n$ female (\%) & $91(55.83)$ \\
Medication use & \\
$\quad$ Anti-epileptic drug use, \% & 36.2 \\
$\quad$ Barbiturate use, \% & 2.45 \\
Benzodiazepine use, \% & 11.04 \\
Antipsychotic use, \% & 9.82 \\
Antidepressant use, \% & 11.04 \\
$\quad$ Other medications, mean (SD, range) & $2.26(2.75,0-13)$ \\
Past medical history & \\
$\quad$ Diagnosis of epilepsy, \% & 40.49 \\
History of stroke, \% & 19.02 \\
Diagnosed degenerative brain disease, \% & 4.91 \\
Diagnosed psychiatric disorder, \% & 12.27 \\
$\quad$ Diagnosed neurodevelopmental disorder, \% & 3.68 \\
Other brain disorder or injury, \% & 16.56 \\
\hline
\end{tabular}

103 EEG preprocessing \& analysis

104 Each subject contributed one EEG recording. For subjects with multiple recordings, the

105 recording corresponding to the physician report containing the most complete clinical

106 picture was selected. For recordings that were split into multiple segments, the longest of

107 the segments was chosen for preprocessing. All preprocessing was performed using the

108 FieldTrip toolbox in MATLAB (www.fieldtriptoolbox.org) (Oostenveld et al., 2011). For each

109 selected recording, 19 scalp electrodes of the International 10-20 system that were common

110 to all subjects were selected. The resulting continuous recordings were segmented into 4-s

111 trials, producing an average of 317 trials per subject, and bandpass filtered (0.5 to $55 \mathrm{~Hz}$ ).

112 The majority of recordings were sampled at $250 \mathrm{~Hz}$, but one subject that was sampled at 512

$113 \mathrm{~Hz}$ was downsampled to $250 \mathrm{~Hz}$ before proceeding. 
Two trial removal steps were then completed. The majority of subjects received

115 photic stimulation. For these subjects, trials where photic stimulation began and ended were

116 detected, and the trials within this range to 5 trials past the end of stimulation were removed.

117 Trials at the beginning of a recording where the amplitude of the photic channel was not zero

118 were also removed. Next, trials with excessive signal amplitude were detected for removal.

119 For each subject, $30 \%$ of the trials that were determined by visual inspection to be

120 reasonably free of artifacts were selected. Global field power was calculated and its mean \pm

$1215 \mathrm{std}$ was used to reject trials with time points outside of this threshold. The average number

122 of remaining trials per subject following both of these removal steps was 178.

123 Independent component analysis was next used to remove ocular and muscle

124 artifacts. Components with topographical distributions typical of these artifacts were

125 selected and their traces further examined. Where possible, probable ocular artifact

126 components were confirmed via alignment of the component trace with the

127 electrooculogram traces from the original recording. Probable muscle artifact components

128 were confirmed by the presence of a high frequency component trace. Finally, any recordings

129 not referenced to a common average were re-referenced.

130 MSE (Costa et al., 2005, 2002) was computed by first coarse-graining the EEG time

131 series of each trial into 20 scales. To produce the time series coinciding with a given scale $t$,

132 data points from the original time series within non-overlapping windows of length $t$ were

133 averaged. Thus scale 1 represents the original time series, with 1000 data points per channel

134 per trial resulting from 4 seconds of recording sampled at $250 \mathrm{~Hz}$. Next, sample entropy was

135 calculated for each time series across all scales. This measured the predictability of the

136 amplitude between two versus three consecutive data points $(\mathrm{m}=2)$, with the condition that 
137 data points were considered to have indistinguishable amplitude from one another if the

138 absolute difference in amplitude between them was $\leq 50 \%$ of the standard deviation of the

139 time series $(r=0.5)$. The resulting values were averaged across trials to produce a single

140 MSE curve per channel for each subject. As an entropy-based measure, MSE values are low

141 for both completely deterministic as well as completely uncorrelated signals.

142 Changes in MSE occur with changes in spectral power (Lippé et al., 2009; McIntosh

143 et al., 2008) so we additionally assessed spectral power (SPD) alongside MSE. SPD was

144 calculated for each trial using the fast Fourier transform with a Hann window. To account

145 for age-related global signal power changes, each recording was first normalized (mean = 0 ,

$146 S D=1$ ). Relative spectral power was then calculated for each trial, and results averaged

147 across trials to acquire mean SPD per channel for each subject.

\section{Partial Least Squares Analysis}

149 MSE and SPD measures were each correlated with the available demographic and clinical

150 data using a Partial Least Squares (PLS) analysis (Krishnan et al., 2011; McIntosh and

151 Lobaugh, 2004). This multivariate statistical approach identifies a set of latent variables

152 (LVs) that represent the maximal covariance between two datasets. First, the correlation

153 between the MSE/SPD and clinical data was computed across subjects. Singular value

154 decomposition was then performed on the correlation matrix to produce LVs, each

155 containing three elements: 1) a set of weighted "saliences" that describe a spatiotemporal

156 brain pattern of MSE/SPD measures; 2) a scalar singular value that expresses the strength of

157 the covariance; and 3) a design contrast of correlation coefficients that express how the

158 clinical data relate to the saliences. The mutually orthogonal LVs are extracted in order of

159 magnitude, whereby the first LV explains the most covariance between MSE/SPD and clinical 
160 data, the second LV the second most, and so forth. The significance of each LV was assessed

161 with permutation testing by randomly reordering subjects' MSE/SPD pairing with clinical

162 data to produce 1000 permuted sets for singular value decomposition, with the set of 1000

163 singular values forming the null distribution. The reliability of the MSE/SPD at each

164 electrode in expressing the covariance pattern of each LV was assessed using bootstrap

165 resampling. A set of 500 bootstrap samples was created by resampling subjects with

166 replacement. The ratio between the saliences and the estimated standard error (bootstrap

167 ratio) was taken as an index of reliability. With the assumption that the bootstrap

168 distribution is normal, the bootstrap ratio is akin to a Z-score and corresponding saliences

169 are considered to be reliable if the absolute value of their bootstrap ratio is $>=2$. For the

170 clinical data, confidence intervals were calculate from the upper and lower bounds of the

$17195^{\text {th }}$ percentile of the bootstrap distribution of the correlation with the scores from the

172 MSE/SPD data. The scores are the dot-product of the saliences with the data for each subject

173 and are similar to a factor score from factor analysis.

$174 \quad$ For the demographic and clinical data entered into the PLS analysis, age and number

175 of non-brain medications were treated as continuous variables, while all other variables

176 were categorical. Sex was coded as $0(\mathrm{~F})$ and $1(\mathrm{M})$. The remaining variables were coded as

1770 (not on drug or does not have condition) or 1 (on drug or has condition). 


\section{Results}

179 To determine whether different and heterogeneous clinical profiles can result in differences

180 in brain signal complexity, MSE curves for each subject were correlated with their

181 demographic and clinical data using a PLS analysis. The singular value decomposition of the

182 correlation matrix resulted in two significant LVs. The first LV showed a differentiation

183 between brain disorders, with a global shift towards greater signal complexity in finer time

184 scales and lower signal complexity in coarser time scales across all electrodes for subjects

185 who experienced generalized seizures or those taking antidepressants as compared to those

186 with other brain conditions (i.e., focal seizures, stroke, neurodevelopmental disorders) or

187 using other medications (i.e., anti-epileptics, barbiturates) (Fig. 1A-B). This shift in MSE was

188 evident when a median-split was performed to classify subjects according to how much they

189 expressed the patterns of the LV (i.e., a median split of the LV-scores, Fig. 1C). This LV was

190 significant $(\mathrm{p}<0.001)$ and accounted for $57.8 \%$ of the covariance in the data.

A

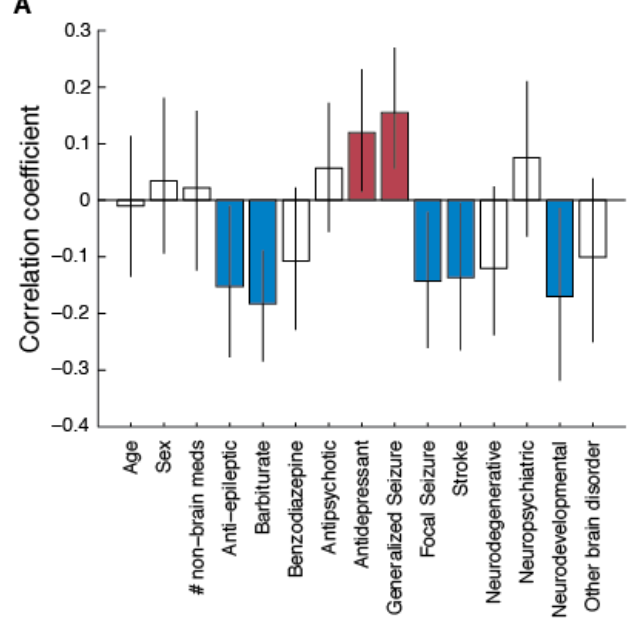

B

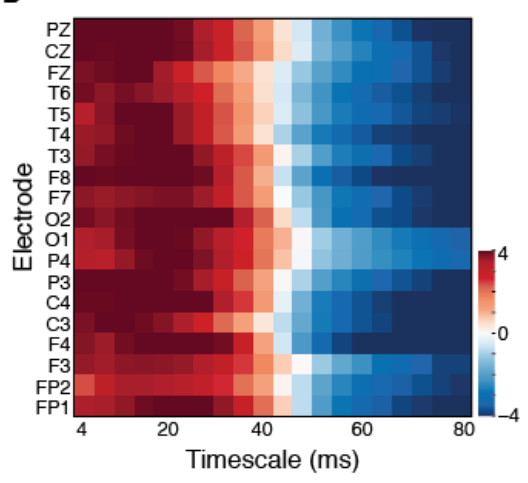

c

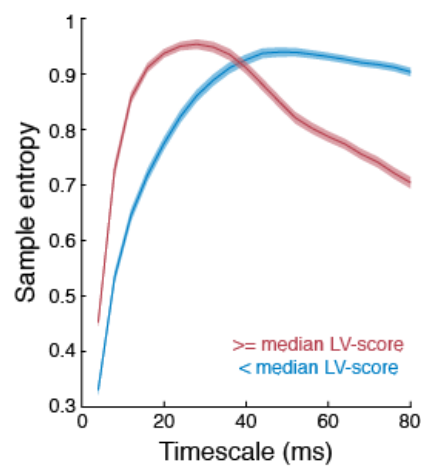

191

192

193

194

195

196
Figure 1. Brain signal complexity differentiates brain disorders. (A) Correlation coefficients and (B) bootstrap ratios of the first latent variable relating clinical data to MSE curves. (C) Average ( \pm SEM) MSE curves, with subjects split into two groups according to their LV-scores. MSE curves were first averaged across electrodes within subjects, then averaged across subjects within each group. In (A), variables whose coefficients are significantly different from 0 are indicated in color for ease of interpretation. 
$201(\mathrm{p}<0.01)$ and accounted for $29.0 \%$ of the covariance in the data.

A

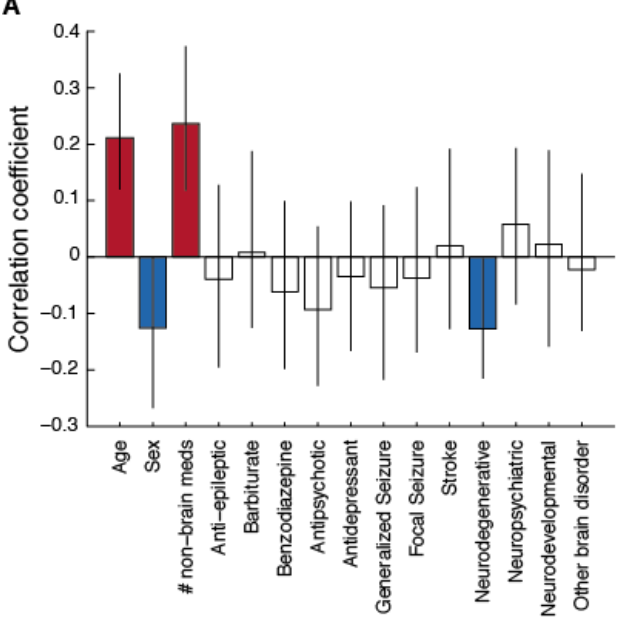

B

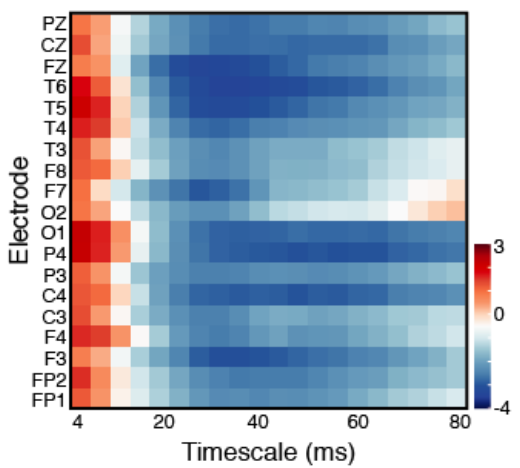

C

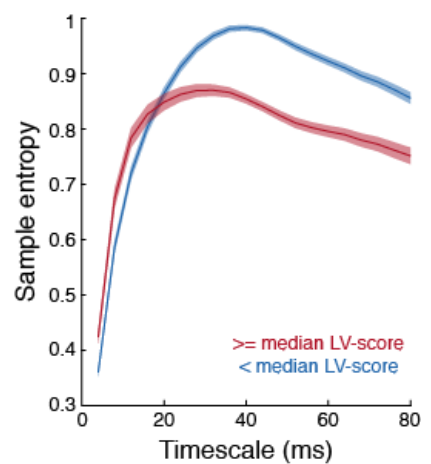

Figure 2. Brain signal complexity differs for older unhealthy males. Correlation coefficients (A) and bootstrap ratios (B) of the second latent variable relating clinical data to MSE curves. (C) Average ( \pm SEM) MSE curves, with subjects split into two groups according to their LV-scores. MSE curves were first averaged across electrodes within subjects, then averaged across subjects within each group. In (A), variables whose coefficients are significantly different from 0 are indicated in color for ease of interpretation.

211 timescale-dependent shift in brain signal complexity associated with different brain and

212 non-brain disorders. A similar analysis of SPD indicated that SPD profiles could only

213 differentiate between subjects with epilepsy from those without epilepsy (Figure 3). 

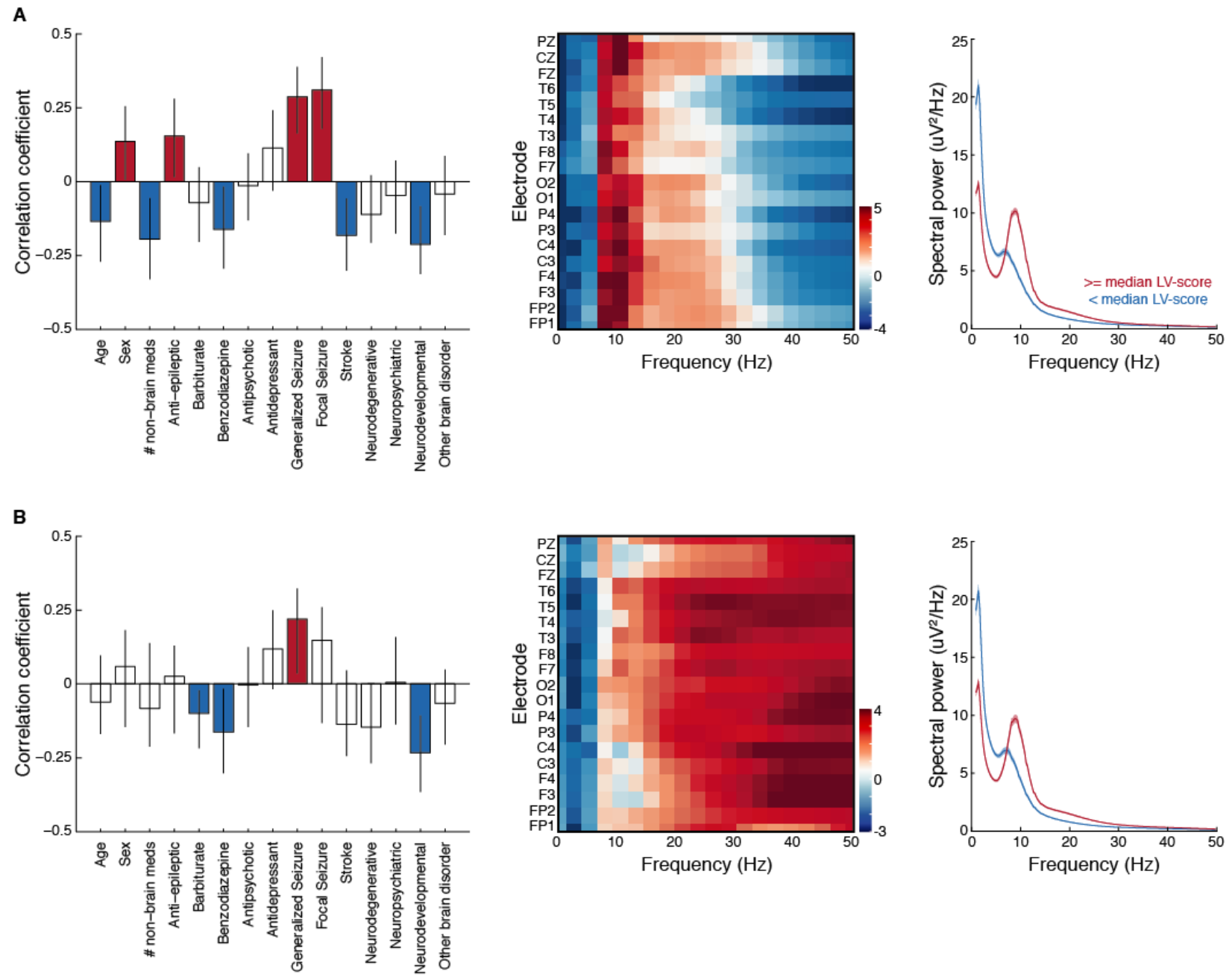

C
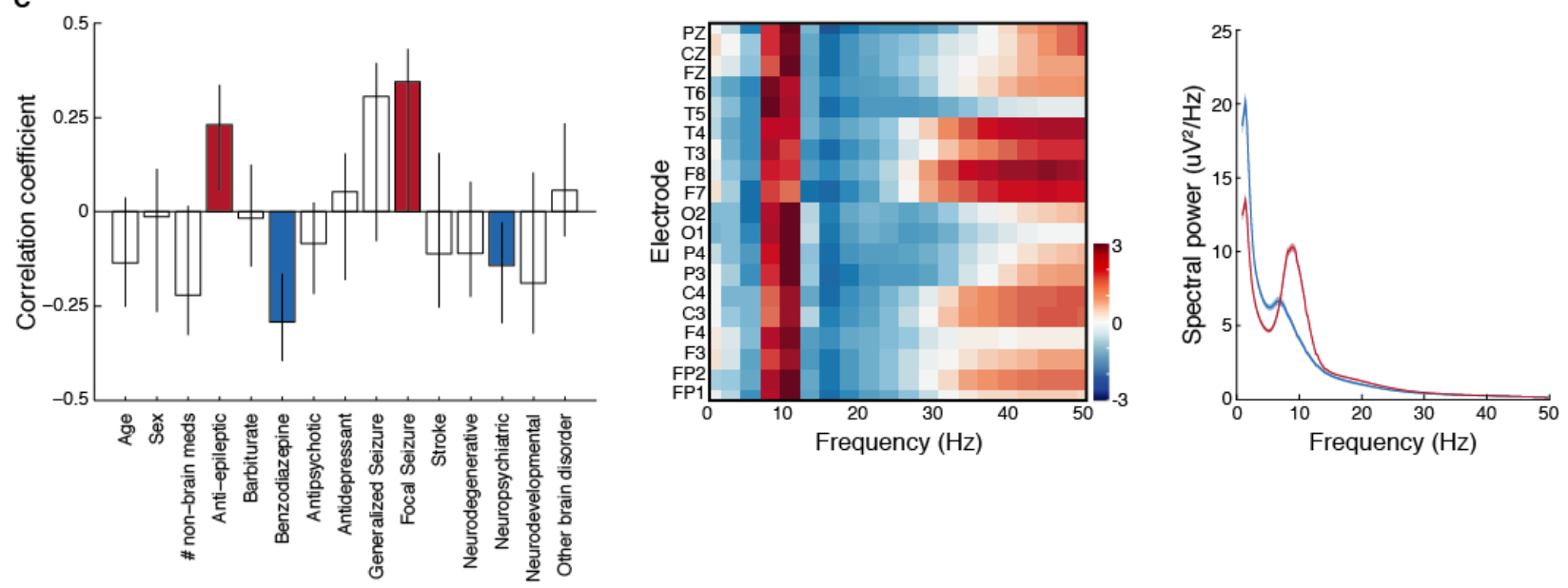

Figure 3. Spectral power density differentiates epilepsy from other brain disorders. (A) First latent variable ( $\mathrm{p}<0.001 ; 50.1 \%$ covariance explained) (B) second latent variable $(\mathrm{p}<0.001 ; 29.9 \%$ covariance explained), and (C) third latent variable ( $\mathrm{p}=0.068 ; 10.1 \%$ covariance explained) of a PLS analysis relating clinical data to SPD. Left panels: correlation coefficients; middle panels: bootstrap ratios; right panels: average $( \pm$ SEM) SPD, with subjects split into two groups according to their LV-scores. SPD functions were first averaged across electrodes within subjects, then averaged across subjects within each group. 


\section{Discussion}

222 In this study, we examined whether brain signal complexity varied across individuals of a

223 large and heterogeneous clinical population using a data driven approach. We found

224 timescale-dependent differences in brain signal complexity for individuals who experience

225 generalized seizures from individuals who have other brain disorders (e.g., focal seizures,

226 stroke, neurodevelopmental disorders). We also found a timescale-dependent shift in brain

227 signal complexity for older males on various medications not related to neurological or

228 neurodegenerative disease that was not evident in the spectral power of the clinical-EEG

229 recordings. Our findings suggest that brain signal complexity, as indexed by MSE, can provide

230 additional insights into brain health status and function not captured by spectral power.

231 In line with the notion that the brain is a dynamical system in which "noise" allows

232 for flexible functioning and a variety of metastable states (Deco et al., 2017, 2011), MSE can

233 be considered as an index of functional repertoire (Heisz et al., 2012). Changes to brain

234 function and dynamics can occur with neurological disease and, indeed, differences in MSE

235 from matched controls have been reported for both epilepsy (Weng et al., 2015) and

236 neurodegenerative disease (Tsai et al., 2015). Here we build on these previous reports by

237 showing how the changes in MSE in these neurological conditions can be differentiated from

238 each other. A complementary data-driven analysis of SPD showed changes in power across

239 frequency bands that differentiated epilepsy from all other diagnoses as well as generalized

240 from focal seizures, consistent with numerous accounts of SPD differences in epilepsy

241 (Clemens et al., 2000; Díaz et al., 1998; Niso et al., 2015; Quraan et al., 2013; Walker, 2008).

242 However, the differentiation of individuals with non-neurological comorbidities was unique

243 to MSE. 
244 The MSE results also replicate previous observations that the scale-dependent changes are

245 indicative of neurodegenerative disorders (Figure 2). Higher MSE at coarse-scales was

246 shown to predict cognitive decline in Parkinson's patients who would develop dementia

247 (Bertrand et al., 2016). The relative balance within subjects between fine and coarse scales

248 also relates to cognitive status in aging (Heisz et al., 2015). These results, considered in the

249 context of the present data, suggest that the relative shifts of complexity across temporal

250 scales may be a sensitive index to assist in clinical evaluation, particular as a predictor of

251 future cognitive decline (McIntosh, 2019).

Metabolic diseases such as diabetes mellitus are known to affect brain structure and

253 cognitive function (Soininen et al., 1992; Tan et al., 2011). More recently, changes to resting-

254 state functional networks have been observed in individuals with diabetes mellitus

255 compared to controls (Y. F. Wang et al., 2016). Autonomic dysfunction, such as hypertension

256 and heart failure, is also a well-documented risk factor for cognitive impairment

257 (Alagiakrishnan et al., 2016; Cannon et al., 2017; Meissner, 2016) and has been associated

258 with changes to brain structure (Kumar et al., 2015; Moon et al., 2018; Suzuki et al., 2017)

259 and function (Bu et al., 2018; Li et al., 2015; Park et al., 2016). As such, both diabetes mellitus

260 and hypertension have been linked to neurological disorders such as stroke (Turin et al.,

261 2016) and dementia (Ninomiya, 2014). One previous report has shown how hypoglycemic

262 conditions in individuals with Type 1 diabetes mellitus results in changes to brain signal MSE

263 (Fabris et al., 2014). We extend these previous findings by showing that the effects of various

264 non-neurological diseases on the brain can be detected by MSE. Together with evidence that

265 MSE changes in response to medical therapies (Farzan et al., 2017; Jaworska et al., 2018; 
266 Liang et al., 2014; Okazaki et al., 2015), MSE offers a promising avenue for the development

267 of clinical biomarkers.

\section{Acknowledgments}

269 This research was supported by a grant from the J. S. McDonnell Foundation to A. R. M.

\section{CRediT Author Statement}

271 Kelly Shen: Conceptualization, Software, Formal Analysis, Validation, Visualization, Writing

272 - Original Draft Alison McFadden: Data Curation, Software, Formal Analysis Anthony R.

273 McIntosh: Conceptualization, Methodology, Writing - Review \& Editing, Supervision,

274 Funding Acquisition

\section{Disclosure Statement}

276 The authors have no competing interests to declare. 


\section{References}

278 Alagiakrishnan, K., Mah, D., Ahmed, A., Ezekowitz, J., 2016. Cognitive decline in heart failure.

Berg, A.T., Berkovic, S.F., Brodie, M.J., Buchhalter, J., Cross, J.H., Van Emde Boas, W., Engel, J.,

French, J., Glauser, T.A., Mathern, G.W., Moshé, S.L., Nordli, D., Plouin, P., Scheffer, I.E.,

Report of the ILAE Commission on Classification and Terminology, 2005-2009.

Bertrand, J.-A., McIntosh, A.R., Postuma, R.B., Kovacevic, N., Latreille, V., Panisset, M.,

Blume, W.T., Lüders, H.O., Mizrahi, E., Tassinari, C., Van Emde Boas, W., Engel, J., 2001.

Bu, L., Huo, C., Xu, G., Liu, Y., Li, Z., Fan, Y., Li, J., 2018. Alteration in Brain Functional and Effective Connectivity in Subjects With Hypertension. Front. Physiol. 9, 669. https://doi.org/10.3389/fphys.2018.00669

Cannon, J.A., Moffitt, P., Perez-Moreno, A.C., Walters, M.R., Broomfield, N.M., McMurray, J.J.V., 
epilepsy syndromes. Epilepsy Res. 42, 105-15.

301 Costa, M., Goldberger, A.L., Peng, C., 2002. Multiscale Entropy Analysis of Complex

302 Physiologic Time Series 6-9. https://doi.org/10.1103/PhysRevLett.89.068102

303 Costa, M., Goldberger, A.L., Peng, C.K., 2005. Multiscale entropy analysis of biological signals.

304 Phys. Rev. E - Stat. Nonlinear, Soft Matter Phys. 71.

306 Deco, G., Jirsa, V.K., McIntosh, A.R., 2011. Emerging concepts for the dynamical organization

307 of resting-state activity in the brain. Nat. Rev. Neurosci. 12, 43-56.

308 https://doi.org/10.1038/nrn2961

309

310

311

312

313

314

315

316

317

318

319

320

321

322

Deco, G., Kringelbach, M.L., Jirsa, V.K., Ritter, P., 2017. The dynamics of resting fluctuations in the brain: metastability and its dynamical cortical core. Sci. Rep. 7, 3095. https://doi.org/10.1038/s41598-017-03073-5

Díaz, G.F., Virués, T., San Martín, M., Ruiz, M., Galán, L., Paz, L., Valdés, P., Díaz, G.., Virués, T., San Martín, M., Ruiz, M., Galán, L., Paz, L., Valdés, P., 1998. Generalized background qEEG abnormalities in localized symptomatic epilepsy. Electroencephalogr. Clin. Neurophysiol. 106, 501-7. https://doi.org/10.1016/s0013-4694(98)00026-1

Fabris, C., Sparacino, G., Sejling, A.-S., Goljahani, A., Duun-Henriksen, J., Remvig, L.S., Juhl, C.B., Cobelli, C., 2014. Hypoglycemia-Related Electroencephalogram Changes Assessed by Multiscale Entropy. Diabetes Technol. Ther. 16, 688-694. https://doi.org/10.1089/dia.2013.0331

Farzan, F., Atluri, S., Mei, Y., Moreno, S., Levinson, A.J., Blumberger, D.M., Daskalakis, Z.J., 2017. Brain temporal complexity in explaining the therapeutic and cognitive effects of seizure therapy. Brain 140, 1011-1025. https://doi.org/10.1093/brain/awx030 
323 Garrett, D.D., Epp, S.M., Perry, A., Lindenberger, U., 2018. Local temporal variability reflects

324 functional integration in the human brain. Neuroimage 183, 776-787.

325 https://doi.org/10.1016/j.neuroimage.2018.08.019

326 Grieder, M., Wang, D.J.J., Dierks, T., Wahlund, L.-O., Jann, K., 2018. Default Mode Network

327 Complexity and Cognitive Decline in Mild Alzheimer's Disease. Front. Neurosci. 12, 770. https://doi.org/10.3389/fnins.2018.00770

Hager, B., Yang, A.C., Brady, R., Meda, S., Clementz, B., Pearlson, G.D., Sweeney, J.A., Tamminga, C., Keshavan, M., 2017. Neural complexity as a potential translational biomarker for psychosis. J. Affect. Disord. 216, 89-99. https://doi.org/10.1016/j.jad.2016.10.016

332 Hasegawa, C., Takahashi, T., Yoshimura, Y., Nobukawa, S., Ikeda, T., Saito, D.N., Kumazaki, H., Minabe, Y., Kikuchi, M., 2018. Developmental Trajectory of Infant Brain Signal Variability: A Longitudinal Pilot Study. Front. Neurosci. 12, 566.

Heisz, J.J., Gould, M., McIntosh, A.R., 2015. Age-related shift in neural complexity related to task performance and physical activity. J. Cogn. Neurosci. 27, 605-13. https://doi.org/10.1162/jocn_a_00725

339 Heisz, J.J., Shedden, J.M., McIntosh, A.R., 2012. Relating brain signal variability to knowledge representation. https://doi.org/10.1016/j.neuroimage.2012.08.018

342 Jaworska, N., Wang, H., Smith, D.M., Blier, P., Knott, V., Protzner, A.B., 2018. Pre-treatment

343 EEG signal variability is associated with treatment success in depression. NeuroImage. 344 Clin. 17, 368-377. https://doi.org/10.1016/j.nicl.2017.10.035

345 Jeste, S.S., Frohlich, J., Loo, S.K., 2015. Electrophysiological biomarkers of diagnosis and 
outcome in neurodevelopmental disorders. Curr. Opin. Neurol. 28, 110-116. https://doi.org/10.1097/WC0.0000000000000181

348 Krishnan, A., Williams, L.J., McIntosh, A.R., Abdi, H., 2011. Partial Least Squares (PLS)

349 methods for neuroimaging: A tutorial and review. Neuroimage 56, 455-475.

350 https://doi.org/10.1016/j.neuroimage.2010.07.034

351 Kumar, R., Yadav, S.K., Palomares, J.A., Park, B., Joshi, S.H., Ogren, J.A., Macey, P.M., Fonarow,

352 G.C., Harper, R.M., Woo, M.A., 2015. Reduced Regional Brain Cortical Thickness in Patients with Heart Failure. PLoS One 10, e0126595.

Li, X., Liang, Y., Chen, Y., Zhang, J., Wei, D., Chen, K., Shu, N., Reiman, E.M., Zhang, Z., 2015.

Liang, W.-K., Lo, M.-T., Yang, A.C., Peng, C.-K., Cheng, S.-K., Tseng, P., Juan, C.-H., 2014.

360 Revealing the brain's adaptability and the transcranial direct current stimulation facilitating effect in inhibitory control by multiscale entropy. Neuroimage 90, 218-234. https://doi.org/10.1016/j.neuroimage.2013.12.048

363 Lippé, S., Kovacevic, N., McIntosh, A.R., 2009. Differential maturation of brain signal

364 complexity in the human auditory and visual system. Front. Hum. Neurosci. 3, 48. https://doi.org/10.3389/neuro.09.048.2009

366 Lu, W.-Y., Chen, J.-Y., Chang, C.-F., Weng, W.-C., Lee, W.-T., Shieh, J.-S., 2015. Multiscale 367 Entropy of Electroencephalogram as a Potential Predictor for the Prognosis of Neonatal 368 Seizures. PLoS One 10, e0144732. https://doi.org/10.1371/journal.pone.0144732 
McIntosh, A.R., 2019. Neurocognitive Aging and Brain Signal Complexity, in: Oxford Research Encyclopedia of Psychology. Oxford University Press, pp. 1-25. https://doi.org/10.1093/acrefore/9780190236557.013.386

McIntosh, A.R., Kovacevic, N., Itier, R.J., 2008. Increased brain signal variability accompanies lower behavioral variability in development. PLoS Comput. Biol. 4. https://doi.org/10.1371/journal.pcbi.1000106

McIntosh, A.R., Lobaugh, N.J., 2004. Partial least squares analysis of neuroimaging data: applications and advances. Neuroimage 23 Suppl 1, S250-63. Spatiotemporal dependency of age-related changes in brain signal variability. Cereb.

Meissner, A., 2016. Hypertension and the Brain: A Risk Factor for More Than Heart Disease. Cerebrovasc. Dis. 42, 255-262. https://doi.org/10.1159/000446082

Mišić, B., Doesburg, S.M., Fatima, Z., Vidal, J., Vakorin, V.A., Taylor, M.J., McIntosh, A.R., 2015. Coordinated Information Generation and Mental Flexibility: Large-Scale Network Disruption in Children with Autism. Cereb. Cortex 25, 2815-2827. https://doi.org/10.1093/cercor/bhu082

Misić, B., Mills, T., Taylor, M.J., McIntosh, A.R., 2010. Brain noise is task dependent and region specific. J. Neurophysiol. 104, 2667-2676. https://doi.org/10.1152/jn.00648.2010

389 Miskovic, V., Owens, M., Kuntzelman, K., Gibb, B.E., 2016. Charting Moment-to-Moment Brain 390 Signal Variability From Early to Late Childhood. Cortex. 83, 51. 391 https://doi.org/10.1016/J.CORTEX.2016.07.006 
Moon, C., Melah, K.E., Johnson, S.C., Bratzke, L.C., 2018. Sleep-disordered breathing, brain volume, and cognition in older individuals with heart failure. Brain Behav. 8, e01029. https://doi.org/10.1002/brb3.1029

Ninomiya, T., 2014. Diabetes Mellitus and Dementia. Curr. Diab. Rep. 14, 487. https://doi.org/10.1007/s11892-014-0487-z

Niso, G., Carrasco, S., Gudín, M., Maestú, F., del-Pozo, F., Pereda, E., 2015. What graph theory actually tells us about resting state interictal MEG epileptic activity. NeuroImage Clin. 8, 503-515. https://doi.org/10.1016/j.nicl.2015.05.008

400 Niu, Y., Wang, B., Zhou, M., Xue, J., Shapour, H., Cao, R., Cui, X., Wu, J., Xiang, J., 2018. Dynamic 401 Complexity of Spontaneous BOLD Activity in Alzheimer's Disease and Mild Cognitive Impairment Using Multiscale Entropy Analysis. Front. Neurosci. 12, 677.

404 Obeid, I., Picone, J., 2016. The Temple University Hospital EEG Data Corpus. Front. Neurosci. 10, 196. https://doi.org/10.3389/fnins.2016.00196

406 Okazaki, R., Takahashi, T., Ueno, K., Takahashi, K., Ishitobi, M., Kikuchi, M., Higashima, M., 407 Wada, Y., 2015. Changes in EEG Complexity with Electroconvulsive Therapy in a Patient 408 with Autism Spectrum Disorders: A Multiscale Entropy Approach. Front. Hum. Neurosci. 9, 106. https://doi.org/10.3389/fnhum.2015.00106

410 Oostenveld, R., Fries, P., Maris, E., Schoffelen, J.-M., 2011. FieldTrip: Open source software for 411 advanced analysis of MEG, EEG, and invasive electrophysiological data. Comput. Intell. Neurosci. 2011, 156869. https://doi.org/10.1155/2011/156869

413 Park, B., Roy, B., Woo, M.A., Palomares, J.A., Fonarow, G.C., Harper, R.M., Kumar, R., 2016. $414 \quad$ Lateralized Resting-State Functional Brain Network Organization Changes in Heart 
Failure. PLoS One 11, e0155894. https://doi.org/10.1371/journal.pone.0155894

416

417

418

Quraan, M.A., McCormick, C., Cohn, M., Valiante, T.A., McAndrews, M.P., 2013. Altered resting state brain dynamics in temporal lobe epilepsy can be observed in spectral power, functional connectivity and graph theory metrics. PLoS One 8, e68609. https://doi.org/10.1371/journal.pone.0068609

Raja Beharelle, A., Kovačević, N., McIntosh, A.R., Levine, B., Beharelle, A.R., Kovačević, N., McIntosh, A.R., Levine, B., Raja Beharelle, A., Kovačević, N., McIntosh, A.R., Levine, B., 2012. Brain signal variability relates to stability of behavior after recovery from diffuse brain injury. Neuroimage 60 , $1528-1537$. https://doi.org/10.1016/j.neuroimage.2012.01.037

Richman, J.S., Moorman, J.R., 2000. Physiological time-series analysis using approximate entropy and sample entropy. Am. J. Physiol. Circ. Physiol. 278, H2039-H2049. https://doi.org/10.1152/ajpheart.2000.278.6.H2039

Sleimen-Malkoun, R., Perdikis, D., Müller, V., Blanc, J.-L., Huys, R., Temprado, J.-J., Jirsa, V.K., 2015. Brain Dynamics of Aging: Multiscale Variability of EEG Signals at Rest and during an Auditory Oddball Task. eNeuro 2. https://doi.org/10.1523/ENEURO.0067-14.2015

Soininen, H., Puranen, M., Helkala, E.L., Laakso, M., Riekkinen, P.J., 1992. Diabetes mellitus and brain atrophy: a computed tomography study in an elderly population. Neurobiol. Aging 13, 717-21.

Suzuki, H., Gao, H., Bai, W., Evangelou, E., Glocker, B., O’Regan, D.P., Elliott, P., Matthews, P.M., 2017. Abnormal brain white matter microstructure is associated with both prehypertension and hypertension. PLoS One 12, e0187600. https://doi.org/10.1371/journal.pone.0187600 
438 Szostakiwskyj, J.M.H., Willatt, S.E., Cortese, F., Protzner, A.B., 2017. The modulation of EEG

439 variability between internally- and externally-driven cognitive states varies with

440 maturation and task performance. PLoS One 12, e0181894.

441 https://doi.org/10.1371/journal.pone.0181894

442 Takahashi, T., 2013. Complexity of spontaneous brain activity in mental disorders. Prog. https://doi.org/10.1016/j.pnpbp.2012.05.001

Takahashi, T., Yoshimura, Y., Hiraishi, H., Hasegawa, C., Munesue, T., Higashida, H., Minabe,

454 Tsai, P.-H., Chang, S.-C., Liu, F.-C., Tsao, J., Wang, Y.-H., Lo, M.-T., 2015. A Novel Application of Middle-Aged Adults. Diabetes Care 34, 1766-1770. https://doi.org/10.2337/dc11Multiscale Entropy in Electroencephalography to Predict the Efficacy of 0308 
https://doi.org/10.1097/HJH.0000000000000753

462 Vakorin, V.A., McIntosh, A.R., 2012. Mapping the multi-scale information content of complex

463 brain signals, in: Rabinovich, M., Friston, K.J., Varona, P. (Eds.), Principles of Brain

464 Dynamics: Global State Interactions. MIT Press, Cambridge, Mass., pp. 183-208.

465 Walker, J.E., 2008. Power Spectral Frequency and Coherence Abnormalities in Patients with

466 Intractable Epilepsy and Their Usefulness in Long-Term Remediation of Seizures Using

467

$$
\text { Neurofeedback. }
$$

Clin.

EEG

Neurosci.

39,

203-205.

Wang, H., McIntosh, A.R., Kovacevic, N., Karachalios, M., Protzner, A.B., 2016. Age-related

Wang, Y.F., Ji, X.M., Lu, G.M., Zhang, L.J., 2016. Resting-state functional MR imaging shed insights into the brain of diabetes. Metab. Brain Dis. 31, 993-1002.

Weng, W.-C., Chang, C.-F., Wong, L.C., Lin, J.-H., Lee, W.-T., Shieh, J.-S., 2017. Altered resting-

Weng, W.-C., Jiang, G.J.A., Chang, C.-F., Lu, W.-Y., Lin, C.-Y., Lee, W.-T., Shieh, J.-S., 2015. Complexity of Multi-Channel Electroencephalogram Signal Analysis in Childhood https://doi.org/10.1007/s11011-016-9872-4 state EEG complexity in children with Tourette syndrome: A preliminary study. Neuropsychology 31, 395-402. https://doi.org/10.1037/neu0000363 
484 in Schizophrenia Characterized by Both Increased Regularity and Randomness.

485 https://doi.org/10.1002/hbm.22763

486 Yang, A.C., Huang, C.-C., Yeh, H.-L., Liu, M.-E., Hong, C.-J., Tu, P.-C., Chen, J.-F., Huang, N.E., Peng,

487 C.-K., Lin, C.-P., Tsai, S.-J., 2013. Complexity of spontaneous BOLD activity in default

488 mode network is correlated with cognitive function in normal male elderly: a multiscale

489 entropy analysis. https://doi.org/10.1016/j.neurobiolaging.2012.05.004 